\title{
Vernetzte Netze leben länger
}

\begin{abstract}
Unternehmensnetzwerke sind nicht nur ein Konstrukt der Organisationswissenschaften, sie spielen in der Praxis eine große Rolle. Zu ihren bekannten Aufgabenstellungen gehören etwa innovative Produktentwicklung, optimierte Wertschöpfung sowie Freisetzung von Synergien. Seit Ende der neunziger Jahre lassen sich zusätzlich verstärkt Netzwerkbildungen beobachten, die die Etablierung nachhaltigen Wirtschaftens in den Regionen als Ziel haben.
\end{abstract}

$\mathrm{D}$ Von Guido Lauen und Michael Schwarz as Leitbild einer nachhaltigen Entwicklung ist als allgemeiner Anspruch und Erfolgsmaßstab kaum noch umstritten. Was aber bedeutet dies für Unternehmen? Allgemein formuliert geht es, um die systematische Verbindung von kurz- und mittelfristigen Zielsetzungen mit den langfristig orientierten Zielen der Existenzsicherung und dem Erhalt der Wettbewerbsfähigkeit um für eine dauerhafte Sicherung von Natur-, Human- und Standortressourcen Sorge zu tragen. Doch ganz offensichtlich fließen die Grundannahmen der Nachhaltigkeit nicht selbstverständlich und reibungslos in praktisches Handeln ein. Noch mangelt es an tragfähigen Brückenschlägen zwischen Zielvorgaben, Umsetzungskonzepten und den Innovationspotenzialen in den relevanten Handlungsfeldern. Eine modellhafte und optimale Umsetzung einer nachhaltigen Entwicklung scheitert an der Konfrontation mit höchst unterschiedlichen Gestaltungsoptionen und -restriktionen, die sich aus vorhandenen Zielkonflikten und inhaltlichen Unvereinbarkeiten zwischen den Vorgaben ökologischer, sozialer und ökonomischer Nachhaltigkeit ergeben.

\section{Innovation durch Kooperation}

Die Verbesserung der unternehmensspezifischen Innovationsfähigkeit in Richtung nachhaltiges Wirtschaften setzt auch eine Ausweitung von unternehmensweiter Kommunikation und Kooperation sowie den Aufbau und die Pflege von Beziehungen zu allen gesellschaftlich relevanten Gruppen und Institutionen am Standort voraus. Denn auch die Unternehmen, die dem Leitbild der Nachhaltigkeit aufgeschlossen gegenüber stehen, sind - auf sich allein gestellt - bei dem Versuch einer praktischen Umsetzung schnell überfordert. Der Übergang zu einer nachhaltigen Wirtschaftsweise scheint ohne eine darauf abgestimmte Kooperation im ganzen Unternehmen und Vernetzung auf unterschiedlichen Ebenen gar nicht machbar zu sein. Dabei ist das gegenseitige Vertrauen der Akteure untereinander - also die Bereitschaft, im Zweifelsfall das Eigeninteresse seines Unternehmens den Gemeinschaftsinteressen des Netzwerkes unterzuordnen - eine erfolgskritische Bedingung.

Sowohl für die Unternehmen als auch für das regionale Umfeld liegen erhebliche Chancen in dem Auf- und Ausbau regionaler Netzwerke für zukunftsfähiges nachhaltiges Wirtschaften. Nachhaltige Zukunftssicherung und Profilierung der Standorte einerseits sowie das Interesse der Unternehmen an innovationsfördernden Rahmenbedingungen und der Minimierung von Reibungsverlusten andererseits können auf diese Weise tendenziell besser in Einklang gebracht werden. So ist nicht verwunderlich, dass sich in den vergangenen Jahren, teilweise unterstïtzt durch Mittel der Deutschen Bundesstiftung Umwelt und des Bundesministeriums für Bildung und Forschung, eine Reihe von regionalen Netzwerken etabliert haben, die sich explizit als Netzwerke nachhaltigen Wirtschaftens verstehen.

\section{Nachhaltigkeitsnetzwerke in der Praxis}

Im Netzwerk ,Nachhaltiges Wirtschaften Heidelberg" bildeten lokale Akteure aus Wirtschaft, Verbänden und Wissenschaft zunächst einen ,Runden Tisch Nachhaltiges Wirtschaften". Neben den ökologischen Zielsetzungen einer Erhöhung der Ressourceneffizienz und Reduzierung der Umweltbelastung wurden ökonomische Aspekte (Kosteneinsparung, Standortsicherung) sowie soziale Nachhaltigkeitskriterien (Chancengleichheit, Verteilungsgerechtigkeit) im Hinblick auf die konkreten Umsetzungsmöglichkeiten in Klein- und Mittelunternehmen diskutiert und ein darauf abgestimmtes praxisorientiertes Schulungs- und Beratungspaket entwickelt. Mit dem Projekt „Nachhaltiges Wirtschaften Heidelberg“ will die
Stadt Heidelberg das Spektrum der kommunalen Wirtschaftspolitik und -förderung erweitern sowie eine nachhaltige Betriebsführung unterstiitzten. Das Kernstïck der Netzwerkarbeit bilden regelmäßige moderierte themenspezifische Workshops mit der Gelegenheit zum Erfahrungsaustausch zwischen den beteiligten Unternehmen. Darüber hinaus beraten externe Sachverständige die Netzwerkunternehmen individuell und schlagen Optimierungen der Betriebsabläufe oder andere Maßnahmen der Ressourcenschonung vor. Am Ende steht der Aufbau eines Umweltmanagementsystems, ergänzt durch Nachhaltigkeitsleitlinien und ein Maßnahmenprogramm.

Dem Netzwerk „COUP 21 - Nürnberger Initiative für Nachhaltiges Wirtschaften" liegt ein über die Hinführung zu Umweltmanagementsystemen hinaus gehendes Nachhaltigkeitsverständnis zu Grunde. Auch hier war das Engagement der Stadtverwaltung von entscheidender Bedeutung. Initiiert durch das Umweltamt der Stadt Nürnberg wurde 1999 das Netzwerk CO-Operation Umweltamt - Pionierunternehmen im 21. Jahrhundert (COUP 21) gegründet. Mitglieder sind Unternehmen, die sich für die Zukunftsfähigkeit der Region Nürnberg engagieren, um im Sinne der lokalen Agenda 21 ökonomische, ökologische, soziale und kulturelle Aspekte der wirtschaftlichen und gesellschaftlichen Entwicklung miteinander zu verbinden. Im Mittelpunkt der Netzwerkarbeit stehen deshalb nicht nur ökologische Fragen, sondern auch Themen wie soziale und interkulturelle Kompetenz, Wissensmanagement oder Familie und Arbeitswelt. Das Netzwerk COUP 21 bietet den Mitgliedsunternehmen eine Plattform zum vertrauensbildenden Erfahrungsaustausch, um auf dem Weg zur Nachhaltigkeit in einer ,,winwin" Struktur voneinander lernen zu können. Über das Akteursnetz zwischen Unternehmen, Behörden, Verbänden, Hochschulen, wissenschaftlichen Instituten und anderen Interessengruppen soll die Effektivität der geplanten Maßnahmen gesteigert werden. Das Umweltamt der Stadt Nürnberg schafft Öffentlichkeit für die ökologischen und sozialen Leistungen der nachhaltigen Unternehmen und übernimmt Aufgaben der Koordination und Moderation.

Auch bei der Gründung des Netzwerks ,,Wuppertaler Umweltinitiative" war die Rolle der Stadt von Ausschlag gebender Bedeutung. Nachdem der Rat der Stadt bereits 1995 beschlossen hatte, aktiv in den Agenda 21-Prozess einzutreten, wurde 2000 ein „Handlungsprogramm Zukunftsfähiges Wuppertal" verabschiedet. Zeitgleich wurde das Netz- 
werk „Wuppertaler Umweltinitiative“ gegründet, deren freiwillige Selbstverpflichtung inzwischen 48 Unterzeichner zählt. Die Stadt Wuppertal praktiziert selbst ein (kommunales) Umweltmanagementsystem und hat sich verpflichtet, im Rahmen des Netzwerkes die Unternehmen bei der Realisierung der oben genannten Ziele im Sinne einer „Hilfe zur Selbsthilfe“ durch „,kundenorientierte und effiziente Dienstleistungen“ einerseits und das Hinwirken auf vereinfachte Regulierungen, Kontrollen und Genehmigungsverfahren andererseits zu unterstïtzen.

Anders als in den zuvor genannten regionalen Nachhaltigkeitsnetzwerken spielen bei „ONNO Ökologisches Unternehmensnetzwerk Ostfriesland“ und dem ,Ulmer Initiativkreis nachhaltige Wirtschaftsentwicklung" Akteure aus dem Bereich der Wissenschaft eine herausragende Rolle - sowohl als Initiatoren der Netzwerke, wie auch als Berater, Promotoren und Netzwerkmanager.

Der „Ulmer Initiativkreis nachhaltige Wirtschaftsentwicklung (unw) “wurde 1993 offiziell ins Leben gerufen. Drei Ziele standen dabei im Vordergrund: erstens für die Ulmer Region zukunftsrelevante Studien über Nachhaltigkeit zu erarbeiten, zweitens nachhaltiges Wirtschaften unter Einbeziehung aller Akteure in der Region und gemäß der auf der RioKonferenz 1992 erarbeiteten und deklarierten Ziele umzusetzen und drittens dabei insbesondere die Unternehmen in der Region einzubeziehen, was sich zum Beispiel in lokalen Agenda 21-Prozessen meist als ein zentrales Hindernis herausgestellt hat. Dem unw wurde ein interdisziplinär zusammengesetzter wissenschaftlicher Beirat zur Seite gestellt, im Zentrum der Netzwerkarbeit stand neben zahlreichen Informations- und Schulungsaktivitäten die Einrichtung und Durchfïhrung mediations-basierter runder Tische. Dieses Instrument wurde sowohl mit Blick auf die unternehmensinterne Kooperation - Mitarbeiter entlang der Wertschöpfungskette erarbeiten gemeinsam nachhaltigkeitsorientierte Lösungen - als auch mit Blick auf die externe Kooperation - nachhaltigkeitsorientierte Zusammenarbeit von Unternehmen - eingesetzt.

Das Netzwerk „ONNO - Ökologisches Unternehmensnetzwerk Ostfriesland“" ist aus einem betriebswirtschaftlichen Projekt der Universität 01denburg hervorgegangen. Hauptziel des Trägervereins ist es, ,,als branchenübergreifendes Netzwerk von ökologisch orientierten Unternehmen, Institutionen, Vereinen und Initiativen (...) ONNO als gemeinnützige Interessenvertretung in Ostfriesland zu etablieren und somit einen Beitrag zur nachhaltigen Regionalentwicklung zu leisten."
Um den Absatz ökologisch und naturnah produzierter Regionalprodukte zu erhöhen, soll die Kooperation von Erzeugern, Verarbeitern und Vermarktern solcher Produkte über Branchengrenzen hinweg gefördert werden. Der zentrale Grundgedanke bei der Vernetzung ist die Maximierung regionaler Wertschöpfung durch enge Stoffströme. Die Einsparpotentiale bei Finanzen und Ressourcen der Mitgliedsunternehmen stehen hier nicht im Vordergrund.

\section{- KMU-Aktivität}

Anders als die bisher beschriebenen Netzwerke rekrutiert sich das „Modell Hohenlohe - Netzwerk betrieblicher Umweltschutz und nachhaltiges Wirtschaften" ausschließlich aus vorwiegend kleinen und mittleren regionalen Produktionsund Dienstleistungsunternehmen, ,die das Rad nicht neu erfinden, sondern gemeinsame Probleme gemeinsam lösen und über den eigenen Tellerrand hinausschauen“ wollen. Seit der Gründung im Jahr 1991 entwickelte sich ein umweltschutz- und nachhaltigkeitsorientiertes Netzwerk, das sich selbst programmatisch wie folgt beschreibt: „Wir wollen: Ökologisch aktiv, sozial engagiert und ökonomisch erfolgreich regionale Kompetenzen aufbauen und nutzen. Wir bieten: Hilfe zur Selbsthilfe für die Umsetzung zukunftsfähiger Lösungen in die betriebliche Praxis. Unsere Mitgliedsbetriebe sind: Mit ihren Mitarbeiterinnen und Mitarbeitern auf dem Weg, die Ziele der nachhaltigen Entwicklung in den Betrieben zu verwirklichen."

Die Unternehmen haben sich als Verein organisiert, dessen Zweck es ist, ,durch Vernetzung vorhandener regionaler/ïberregionaler Kompetenzen das nachhaltige Wirtschaften, das heißt das gleichrangige Beachten ökologischer, ökonomischer und sozialer Belange, sowie das Umweltbewusstsein und die Umweltverantwortung in der Wirtschaft zu fördern und den betrieblichen Umweltschutz zu verbessern. "Dazu soll der überbetriebliche Erfahrungsaustausch in branchen- oder sachspezifischen Arbeitsgruppen institutionalisiert und verstetigt werden. Anders als bei anderen hier vorgestellten Netzwerken wird mit nach der Anzahl der Beschäftigten gestaffelten Beträgen der Mitgliedsunternehmen eine Geschäftsstelle zur Koordination der Netzwerkarbeit finanziert.

Ein besonderen Fall ist das „Kompetenzzentrum Nachhaltiges Wirtschaften Leipzig“, da hier zwar im Rahmen einer Machbarkeitsstudie der Grundstein für ein solides Netzwerk gelegt werden konnte, bislang aber noch keine arbeitsfähigen
Strukturen aufgebaut werden konnten. Die Hauptakteure in dem Prozess der Netzwerkbildung sind das Haus der Umwelt Leipzig, die Stadt und das Umweltforschungszentrum Leipzig. Im Mittelpunkt steht dabei die Absicht, eine standortpolitisch bedeutsame Clusterung mit Nachhaltigkeitsaspekten so zu verbinden, dass die entstehenden Strukturen der lokalen Ökonomie Impulse geben und auch Wissens- und Kompetenztransfer mit den mittel- und osteuropäischen Staaten zur Folge haben.

In einem vier-Stufen-Modell soll die Netzwerkbildung vorangetrieben werden: auf der ersten Stufe sollen etablierte Strukturen in der Region genutzt werden, um Arbeitstreffen zu ermöglichen, auf denen nachhaltigkeitsrelevante Themen gefunden und angegangen werden können. Auf der zweiten Stufe sollen Fachpartner und Entscheider aus den Teilnehmerinstitutionen mit weiteren Interessenten zusammengefuihrt werden, um verbindlich Aufgaben und Inhalte zu definieren. Auf der dritten Stufe sollen Kompetenzen im Themenfeld Nachhaltiges Wirtschaften in dem dann zu gründenden Kompetenzzentrum erarbeitet und transportiert werden. Auf der vierten Stufe soll dann der geschaffene ,nachhaltige Mehrwert" allen Teilnehmern zur Verfügung gestellt werden, um darauf basierend Ausgangspunkte für weitere Qualitätsstufen der Netzwerkarbeit bereitzustellen.

\section{Netzwerke der Nachhaltigkeit nachhaltig vernetzen}

Was kann man aus den Erfahrungen der beschriebenen regionalen Nachhaltigkeitsnetzwerke für eine zukünftige Netzwerkbildung und ein entsprechendes Netzwerkmanagement lernen?

Fast alle untersuchten Netzwerke, mit Ausnahme des Modells Hohenlohe, teilen die Erfahrung, dass nach Ablauf der Förderung (Heidelberg, Ulm, Nürnberg, Leipzig, Ostfriesland) oder nach einer euphorischen Startphase ohne Förderung (Wuppertal) die operative Netzwerkarbeit nach innen und die Öffentlichkeitsarbeit nach außen erlahmt und auf niedrigem Niveau stagniert oder mit tendenziellen Auflösungserscheinungen konfrontiert ist. Mit anderen Worten: Die Netzwerke der Nachhaltigkeit sind weder intern noch netzwerkuibergreifend nachhaltig vernetzt.

Dafür sind primär folgende Gründe ausschlaggebend:

- Die Nutzenerwartungen der teilnehmenden Institutionen sind unklar, unrealistisch und/oder werden im Prozess der Netzwerkbildung nicht definiert beziehungsweise korrigiert. 
- So verwundert es auch nicht, dass nach einer motivierenden Startphase einige Akteure - insbesondere die Leitungsebene der beteiligten Unternehmen - fehlenden (betriebswirtschaftlich fassbaren) Nutzen der Netzwerkteilnahme reklamieren.

- Divergierende Interessen werden nicht thematisiert und bleiben dementsprechend unïberbrückt.

- Damit eng verbunden ist eine asymmetrisch verteilte Bereitschaft, sich für die Belange des Netzwerkes einzusetzen. Beide Mängel verweisen auf die prinzipielle Bedeutung einer vertrauensvollen Zusammenarbeit im Netzwerk, die gegebenenfalls die Relativierung eigener Interessen ermöglicht.

- Innerhalb der teilnehmenden Institutionen werden nur bestimmte Personen und Funktionsträger angesprochen und operativ involviert, ohne zu berücksichtigen, dass sich die Leitungsebene für die notwendige Verbindlichkeit eindeutig und verlässlich positionieren muss.

- Fehlen verbindliche Übereinkünfte zu strittigen Punkten und lassen sich diese in kommunikativen Prozessen nicht herstellen, bleiben die Netzwerke instabil und haben wenig Aussicht, den verfolgten Zielen nahe zu kommen.

- Die Erfahrungen zeigen, dass eine erfolgreiche Netzwerkarbeit ohne ein intensives, vorausschauendes und damit zeitintensives Netzwerkmanagement nicht in Gang kommt. Daraus folgt, dass auch nach einer eventuellen Förderphase Ressourcen für die Schaffung oder Aufrechterhaltung einer zentralen Anlauf- oder so- gar Geschäftsstelle mit angemessener personeller und infrastruktureller Ausstattung bereitgestellt werden müssen.

Wie der vom Nürnberger Netzwerk Coup 21 veranstaltete Nachhaltigkeitskongress unlängst gezeigt hat, konnten dort - aber auch in den anderen Netzwerken - zum Teil beachtliche Erfolge im Sinne einer nachhaltigen Profilierung im Wettbewerb der Regionen realisiert werden. Andererseits hat sich aber auch gezeigt, dass die nachhaltigkeitsorientierte Vernetzung von Unternehmen und anderen relevanten Institutionen, Organisationen und Akteursgruppen, die praktische Nutzung der damit verbundenen Innovationspotenziale sehr hohe Anforderungen an die Koordination und das Netzwerkmanagement stellt, stark von regionalspezifischen Besonderheiten geprägt ist und sich keineswegs im Selbstlauf als Erfolgsmodell konsolidiert und weiterentwickelt. Weitgehend unklar sind insbesondere die im Einzelnen realisierten beziehungsweise realisierbaren Netzwerkeffekte auf Seiten der beteiligten Unternehmen, obwohl dies der entscheidende Hebel zur Motivation für eine aktive Beteiligung ist.

Eine „nachhaltige“ Konsolidierung, Weiterentwicklung und Verbreitung von derartigen nachhaltigkeitsorientierten regionalen Unternehmensnetzwerken hängt entscheidend davon ab, inwieweit es gelingt,

- nach innen und nach außen hin den Nutzen für die Beteiligten, die Zukunftsfähigkeit der Unternehmen und der Region konkret nachvollziehbar zu machen,
- die dafür ausschlaggebenden Erfolgsfaktoren und Netzwerkeffekte zu dokumentieren,

- Themenfelder sowie Handlungshilfen zu entwickeln, die an den vorhandenen Innovationsbedarfen und -potentialen der Unternehmen anknüpfen, sowie

- die Erfahrungen aus anderen Regionen im Hinblick auf eine situationsspezifisch angepasste Übertragbarkeit auszuwerten und einen daraufhin orientierten wechselseitigen Lernprozess und Erfahrungsaustausch zu organisieren.

Ziel eines vom ISO Institut in enger Kooperation mit den genannten Netzwerken seit längerem geplanten Projektes ist es, die Konsolidierungsperspektiven nachhaltigkeitsorientierter regionaler Unternehmensnetzwerke erfahrungsgestützt in diesem Sinne zu optimieren. Bleibt zu hoffen, dass diese gemeinsame Initiative aus Wissenschaft und Praxis die notwendige Unterstützung und Förderung erfährt.

\section{Die Autoren}

Guido Lauen ist wissenschaftlicher Mitarbeiter im Fachbereich Bildungswissenschaften an der Universität Wuppertal. Dr. Michael Schwarz ist Geschäftsführer am ISO Institut zur Erforschung sozialer Chancen.

Kontakt: Uni Wuppertal, Gaußstr. 20, 42097 Wup pertal. Tel. 0202 439-2328, E-Mail: lauen@uniwuppertal.de, ISO Institut, Neusser Str. 219, 50733 Köln. Tel. 0221-88811208, E-Mail: michael.schwarz@iso-koeln.de

\section{oekom verlag - Wir publizieren Zukunft}

\section{UnternehmensGrün (Hrsg.) \\ Mit neuer Energie in die Zukunft \\ Unternehmen zwischen Billigstrom- und Öko-Angeboten}

Billigstrom oder Ökostrom? Kostensenkung oder nachhaltiges Handeln? Diesen Fragen müssen sich auch Unternehmen stellen. Im Zuge der Liberalisierung ist die Monopolstellung der regionalen Stromanbieter aufgehoben. Große Energieversorgungsunternehmen locken mit Billigangeboten. Doch auch den Ökostromanbietern bietet die Öffnung des Marktes Chancen sich zu etablieren. Wie entwickelt sich der ökologische Energiemarkt? Welche Perspektiven eröffnen sich? Wer profitiert? Experten aus Wissenschaft, Politik und Wirtschaft informieren über die vielfältigen Chancen einer ökologischen Energiezukunft und berichten über Beispiele aus der Praxis.

oekom verlag; München 2000; 168 Seiten; 12,80 € (zzgl.Versandkosten im Ausland); ISBN 3-928244-66-3

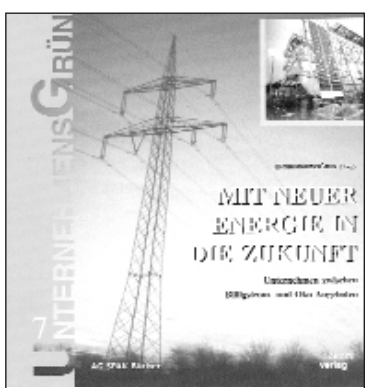

Erhältlich bei wmi verlagsservice $\mathrm{GmbH}$ \& Co. KC,

Auslieferung oekom verlag, 86899 Landsberg am Lech

Fon $++49 /(0) 8191 / 125-249$, Fax -405,

E-Mail f.cengiz@wmi-verlagsservice.de 
(c) 20I0 Authors; licensee IÖW and oekom verlag. This is an article distributed under the terms of the Creative Commons Attribution Non-Commercial No Derivates License (http://creativecommons.org/licenses/by-nc-nd/3.o/), which permits unrestricted use, distribution, and reproduction in any medium, provided the original work is properly cited. 